\title{
Radiation Effects on Unsteady Free Convection Flow Past a Vertical Plate with Newtonian Heating
}

\author{
S. Das \\ Department of Mathematics \\ University of Gour Banga, \\ Malda 732 103, India \\ C. Mandal \\ Department of Applied Mathematics \\ Vidyasagar University, \\ Midnapore 721 102, India
}

\author{
R. N. Jana \\ Department of Applied Mathematics \\ Vidyasagar University, \\ Midnapore 721 102, India
}

\begin{abstract}
The unsteady free convection flow with thermal radiation past a vertical plate with Newtonian heating has been studied. The governing equations have been solved numerically by the implicit finite difference method of Crank- Nicolson's type. The variations of the fluid velocity and temperature are presented graphically. It is found that the fluid velocity decreases near the plate and it increases away from the plate with an increase in either Prandtl number or radiation parameter. It is also found that the fluid velocity increases with an increase in either Grashof number or time. An increase in either Prandtl number or radiation parameter leads to fall in the fluid temperature. Further, it is seen that the shear stress at the plate decreases with an increase in either radiation parameter or Prandtl number. The rate of heat transfer decreases with an increase in Prandtl number for fixed values of radiation parameter and time while it increases with an increase in either radiation parameter or time.
\end{abstract}

\section{Keywords}

Free convection, Radiative heat transfer, Prandtl number, Grashof number and Newtonian heating.

\section{INTRODUCTION}

In the context of space technology and in processes involving high temperatures the effects of radiation are of vital importance. Recent developments in hypersonic flights, missile reentry, rocket combustion chambers, power plants for inter planetary flight and gas cooled nuclear reactors, have focused attention on thermal radiation as a mode of energy transfer and emphasize the need for improved understanding of radiative transfer in these process. The radiative heat transfer is basically very important in many aspects of practical engineering. Examples are the solar radiation in buildings, foundry engineering and solidification processes, chemical engineering, composite structures applied in industry. The radiative heat transfer problems are also encountered in industrial textiles, textiles designed for use under hermetic protective barrier, multilayer clothing materials and needle heating in heavy industrial sewing as explained by Korycki [1]. These are conjugate heat transfer processes, where Newtonian heating finds its engineering applications. Manifestly, high temperature phenomena cannot be ignored. Therefore, it is more realistic to study the problem of unsteady free convection flow with radiative heat transfer past a vertical plate with Newtonian heating. Newtonian heating, where the rate of heat transfer from the bounding surface with a finite heat capacity is proportional to the local surface temperature and is usually termed conjugate convective flow. This configuration occurs in many important engineering devices, for example, in heat exchangers where the conduction in solid tube wall is greatly influenced by the convection in the fluid flowing over it. Literature concerning this subject can be found in books by Hottel and Sarofim [2] and Brewster [3]. Cess [4] has made a complementary study of the interaction of thermal radiation with free convection heat transfer. The natural convection boundary-layer flow on a vertical surface with Newtonian heating has been studied by Merkin [5]. Hossain and Takhar [6] have studied the radiation effect on mixed convection along a vertical plate with uniform surface temperature. The free convection boundary layer flow along a vertical surface in a porous medium with Newtonian heating have been investigated by Lesnic et al. [7]. Muthucumaraswamy and Ganesan [8] have studied the radiation effects on the flow past an impulsively started infinite vertical plate with variable temperature. An exact solution of the unsteady free-convection boundary-layer flow past an impulsively started vertical surface with Newtonian heating have been explained by Chaudhary and Jain $[9,10]$. Mebine and Adigio [11] have investigated the unsteady free convection flow with thermal radiation past a vertical porous plate with Newtonian heating. The radiation effects on free convection near a moving vertical plate with Newtonian heating have been analyzed by Narahari and Ishak [12].

In this present paper, we have studied the unsteady free convection flow with radiative heat transfer over an infinitely long vertical plate when at time $t \leq 0$, both the fluid and plate are at rest with constant temperature $T_{\infty}$. At time $t>0$, the plate is given an impulsive motion in the vertically upward direction against gravitational field with a uniform velocity $U_{0}$. It is found that the fluid velocity $u$ decreases near the plate and it increases away from the plate with an increase in either Prandtl number $P r$ or radiation parameter $R a$. The fluid velocity increases with an increase in either Grashof number $G r$ or time ${ }^{\tau}$. An increase in either Prandtl number $P r$ or radiation parameter $R a$ leads to fall in the fluid temperature $\theta$. Further, it is found that the shear stress $\tau_{x}$ at the plate $(\eta=0)$ decreases with an increase in either radiation parameter $R a$ or Prandtl number $P r$. The rate of heat transfer $-\left(\frac{d \theta}{d \eta}\right)_{\eta=0}$ decreases with an increase in either Prandtl 
number or $R a \operatorname{Pr}$ for fixed value time $\tau$ while it increases with an increase in $\tau$ for for fixed values of Prandtl number $\mathrm{Pr}$ and radiation parameter $R a$.

\section{FORMULATION OF THE PROBLEM AND ITS SOLUTIONS}

Consider an unsteady free convection flow with radiative heat transfer of a viscous incompressible fluid past an impulsively started infinitely long vertical plate with Newtonian heating. The $x$-axis is taken along the vertical plate in an upward direction and $y$-axis is taken normal to the plate (see Fig.1). At time $t \leq 0$, both the fluid and plate are at rest with constant temperature $T_{\infty}$. At time $t>0$, the plate is given an impulsive motion in the vertically upward direction against gravitational field with a characteristic velocity $U_{0}$. It is assumed that the rate of heat transfer from the surface is proportional to the local surface temperature $T$. Since the plate is infinitely long along $x$-direction, all the physical variables are the functions of $y$ and $t$ only. The flow is considered optically thin gray gas with natural convection and radiation. The radiative heat flux in the $x$ - direction is considered negligible in comparison to $y$ direction. The Rosseland approximation is used to describe the radiative heat flux in the energy equation.

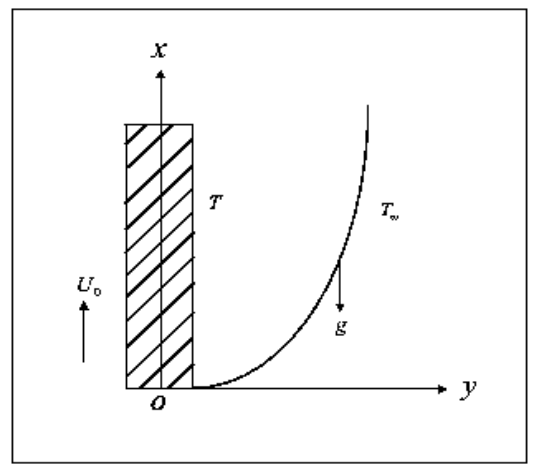

Fig.1: Geometry of the problem.

Under usual Boussinesq approximation, the momentum and energy equations are

$$
\begin{aligned}
& \frac{\partial u^{\prime}}{\partial t}=v \frac{\partial^{2} u^{\prime}}{\partial y^{2}}+g \beta\left(T-T_{\infty}\right), \\
& \rho c_{p} \frac{\partial T}{\partial t}=k \frac{\partial^{2} T}{\partial y^{2}}-\frac{\partial q_{r}}{\partial y},
\end{aligned}
$$

where $u^{\prime}$ is the velocity in the $x$-direction, $T$ the temperature of the fluid, $g$ the acceleration due to gravity, $\beta$ the coefficient of thermal expansion, $v$ the kinematic coefficient of viscosity, $\rho$ the fluid density, $k$ the thermal conductivity, $c_{p}$ the specific heat at constant pressure and $q_{r}$ the radiative heat flux. The heating due to viscous dissipation is neglected for small velocities in the energy equation (2).

The initial and boundary conditions are

$$
u^{\prime}=0, T=T_{\infty} \text { for } y \geq 0 \text { and } t \leq 0,
$$

$$
\begin{aligned}
& u^{\prime}=U_{0}, \frac{\partial T}{\partial y}=-\frac{q}{k} T \text { at } y=0 \text { for } t>0, \\
& u^{\prime} \rightarrow 0, T \rightarrow T_{\infty} \text { as } y \rightarrow \infty \text { for } t>0 .
\end{aligned}
$$

It has been shown by Cogley et al.[13] that in the optically thin limit for a non-gray gas near equilibrium, the following relation holds

$$
\frac{\partial q_{r}}{\partial y}=4\left(T-T_{\infty}\right) \int_{0}^{\infty} K_{\lambda_{0}}\left(\frac{\partial e_{\lambda p}}{\partial T}\right)_{0} d \lambda,
$$

where $K_{\lambda}$ is the absorption coefficient, $\lambda$ is the wave length, $e_{\lambda p}$ is the Plank's function and subscript ' 0 ' indicates that all quantities have been evaluated at the temperature $T_{\infty}$ which is the temperature of the plate at time $t \leq 0$. Thus, our study is limited to small difference of plate temperature to the fluid temperature.

On the use of the equation (4), equation (2) becomes

$$
\rho c_{p} \frac{\partial T}{\partial t}=k \frac{\partial^{2} T}{\partial y^{2}}-4\left(T-T_{\infty}\right) I,
$$

where

$$
I=\int_{0}^{\infty} K_{\lambda_{0}}\left(\frac{\partial e_{\lambda p}}{\partial T}\right)_{0} d \lambda .
$$

Introducing non-dimensional variables

$$
\eta=\frac{y U_{0}}{v}, \tau=\frac{t U_{0}^{2}}{v}, u=\frac{u^{\prime}}{U_{0}}, \theta=\frac{\left(T-T_{\infty}\right)}{T_{\infty}},
$$

equations (1) and (5) become

$$
\begin{aligned}
& \frac{\partial u}{\partial \tau}=\frac{\partial^{2} u}{\partial \eta^{2}}+G r \theta, \\
& \frac{\partial \theta}{\partial \tau}=\frac{1}{\operatorname{Pr}} \frac{\partial^{2} \theta}{\partial \eta^{2}}-\operatorname{Ra} \theta,
\end{aligned}
$$

where $R a=\frac{4 I T_{\infty}}{k}$ is the radiation parameter, $\operatorname{Pr}=\frac{\rho v c_{p}}{k}$, the Prandtl number, $G r=\frac{g \beta v T_{\infty}}{U_{0}^{3}}$, the Grashof number and the characteristic velocity $U_{0}$ is defined by $\frac{q v}{k}$.

The corresponding boundary conditions for $u$ and $\theta$ are

$$
\begin{aligned}
& u=0, \theta=0 \text { for } \eta \geq 0 \text { and } \tau \leq 0, \\
& u=1, \frac{d \theta}{d \eta}=-(1+\theta) \text { at } \eta=0 \text { for } \tau>0, \\
& u \rightarrow 0, \theta \rightarrow 0 \text { as } \eta \rightarrow \infty \text { for } \tau>0 .
\end{aligned}
$$

\section{NUMERICAL SOLUTION}

One of the most commonly used numerical methods is the finite difference technique, which has better stability characteristics, and is relatively simple, accurate and efficient Another essential feature of this technique is that it is based on an iterative procedure and a tridiagonal matrix manipulation. This method provides satisfactory results but it may fail when applied to problems in which the differential equations are very sensitive to the choice of initial conditions. In all numerical solutions the continuous partial differential equation is replaced with a discrete approximation. In this context the word discrete means that the numerical solution is known only at a finite number of points in the physical domain. The number of those points can be selected by the user of the numerical method. In 
general, increasing the number of points not only increases the resolution but also the accuracy of the numerical solution. The discrete approximation results in a set of algebraic equations that are evaluated (or solved) for the values of the discrete unknowns. The mesh is the set of locations where the discrete solution is computed. These points are called nodes and if one were to draw lines between adjacent nodes in the domain the resulting image would resemble a net or mesh.

When time-accurate solutions are important, the CrankNicolson scheme has significant advantages. The CrankNicolson scheme is not significantly more difficult to implement and it has a temporal truncation error that is $O\left(\Delta \tau^{2}\right)$ as explained by Recktenwald [14]. The CrankNicolson scheme is implicit, it is also unconditional stable [15, $16,17]$. In order to solve the equations (8) and (9) under the initial and boundary conditions (10), an implicit finite difference scheme of Crank-Nicolson's type has been employed. The right hand side of the equations (8) and (9) is approximated with the average of the central difference scheme evaluated at the current and the previous time step. The finite difference equation corresponding to equations (8) and (9) are

$$
\begin{aligned}
& \frac{u_{i, j+1}-u_{i, j}}{\Delta \tau} \\
& =\left(\frac{u_{i-1, j}-2 u_{i, j}+u_{i+1, j}+u_{i-1, j+1}-2 u_{i, j+1}+u_{i+1, j+1}}{2(\Delta \eta)^{2}}\right) \\
& +G r\left(\frac{\theta_{i, j+1}+\theta_{i, j}}{2}\right), \\
& \frac{\theta_{i, j+1}-\theta_{i, j}}{\Delta \tau} \\
& =\frac{1}{\operatorname{Pr}}\left(\frac{\theta_{i-1, j}-2 \theta_{i, j}+\theta_{i+1, j}+\theta_{i-1, j+1}-2 \theta_{i, j+1}+\theta_{i+1, j+1}}{2(\Delta \eta)^{2}}\right) \\
& -\operatorname{Ra}\left(\frac{\theta_{i, j+1}+\theta_{i, j}}{2}\right) .
\end{aligned}
$$

The boundary conditions (10) become

$$
\begin{aligned}
& u_{i, 0}=0, \theta_{i, 0}=0 \text { for all } i \neq 0, \\
& u_{0, j}=1, \frac{\theta_{1, j}-\theta_{0, j}}{\Delta \eta}=-\left(1+\theta_{0, j}\right), \\
& u_{N, j}=0, \theta_{N, j}=0,
\end{aligned}
$$

where $N$ corresponds to $\infty$. Here the suffix $i$ corresponds to $\eta$ and $j$ corresponds to $\tau$. Also $\Delta \tau=\tau_{j+1}-\tau_{j}$ and $\Delta \eta=\eta_{i+1}-\eta_{i}$. Knowing the values of $\theta$ and $u$ at a time $\tau$ we can calculate the values at a time $\tau+\Delta \tau$ as follows. We put $i=1,2, \ldots, N-1$, in equation (12) which constitutes a tridiagonal system of equations, the system can be solved by Thomas algorithm as discussed in Carnahan et al.[18]. Thus, $\theta$ is known for all values of $\eta$ at time $\tau$. Then knowing the values of $\theta$ and applying the same procedure with the boundary conditions, we compute $u$ from the equation (11). This procedure is continued to obtain the solution till desired time $\tau$. The Crank-Nicolson scheme has a truncation error of $O\left(\Delta \tau^{2}\right)+O\left(\Delta \eta^{2}\right)$, i.e. the temporal truncation error is significantly smaller.

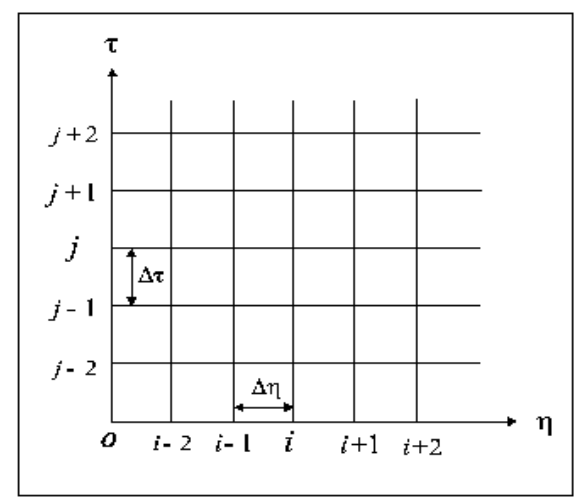

\section{Fig.2: Finite difference grids}

The implicit method gives stable solutions and requires matrix inversions which we did at step forward in time because this problem is an initial -boundary value problem with a finite number of spatial grid points. Though, the corresponding difference equations do not automatically guarantee the convergence of the mesh $\Delta \eta \rightarrow 0$. To achieve maximum numerical efficiency, we used the tridiagonal procedure to solve the two point conditions governing the main coupled governing equations of momentum and energy. The convergence of the process is quite satisfactory and the numerical stability of the method is guaranteed by the implicit nature of the numerical scheme. Hence, the scheme is consistent. Stability and consistency ensure convergence.

\section{RESULTS AND DISCUSSION}

We have presented the non-dimensional fluid velocity $u$ and temperature $\theta$ for several values of the radiation parameter $R a$, Prandtl number $P r$, Grashof number $G r$ and time $\tau$ in Figs.3-9. It is seen from Figs. 3 and 4 that the fluid velocity $u$ decreases near the plate and increases away from the plate with an increase in either Prandtl number $\mathrm{Pr}$ or radiation parameter $R a$. This is due to the fact that the fluids with high Prandtl number have greater viscosity, which makes the fluid thick and hence move slowly. It is also seen that the fluid velocity $u$ is maximum near the plate and decreases away from the plate and finally becomes zero for all values of radiation parameter $R a$ and Prandtl number $\mathrm{Pr}$. Fig.5 shows that an increase in Grashof number $G r$ leads to rise the velocity $u$. This implies that the bouyancy force accelerates the velocity field. It is revealed from Fig. 6 that the fluid velocity $u$ increases with an increase in time $\tau$. Figs. 7 and 8 illustrate that the fluid temperature $\theta$ decreases with an increase in either Prandtl number $\operatorname{Pr}$ or radiation parameter $R a$. The fluid temperature is maximum near the plate and decreases away from the plate and finally takes zero value for all values of radiation parameter $R a$ and Prandtl number $P r$. It is observed from Fig.9 that the fluid temperature increases with an increase in time $\tau$. 


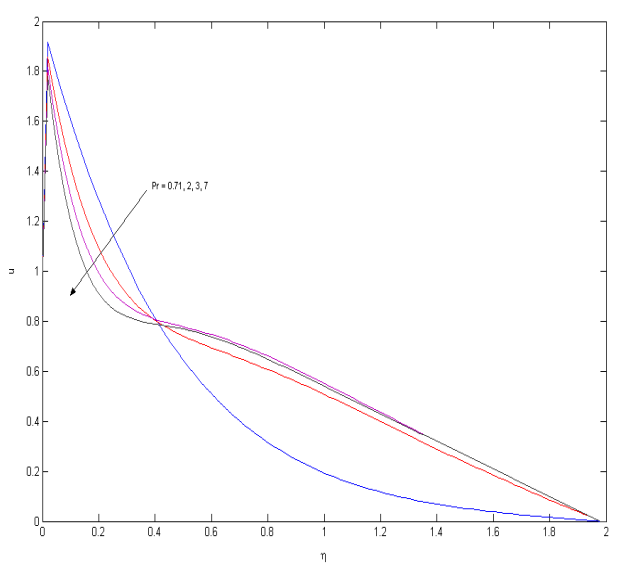

Figure 3: Velocity profiles for $\operatorname{Pr}$ when $G r=5, R a=5$ and $\tau=0.2$

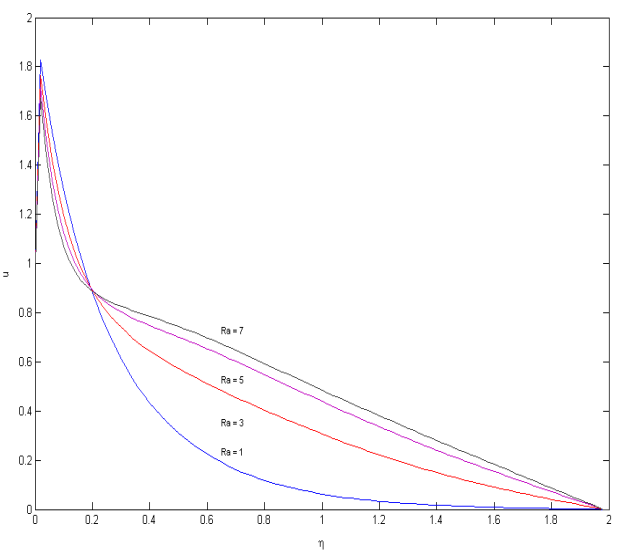

Figure 4: Velocity profiles for $R a$ when $P r=7, G r=5$ and $\tau=0.2$

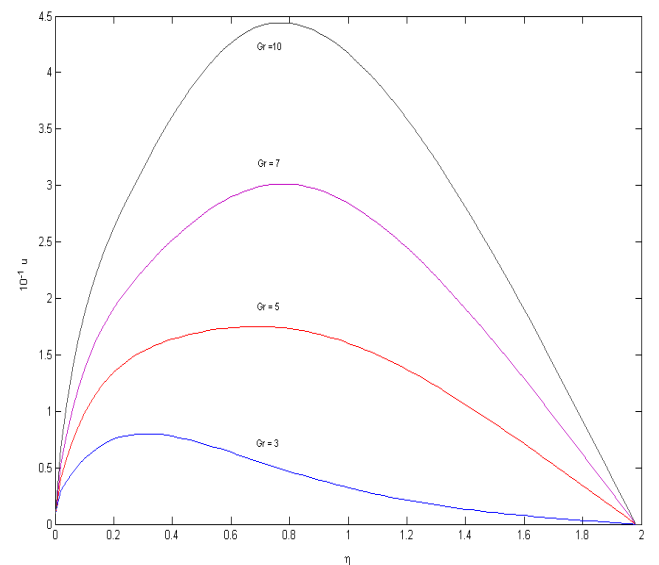

Figure5: Velocity profiles for $G r$ when $R a=1$, $\operatorname{Pr}=0.71$ and $\tau=0.2$

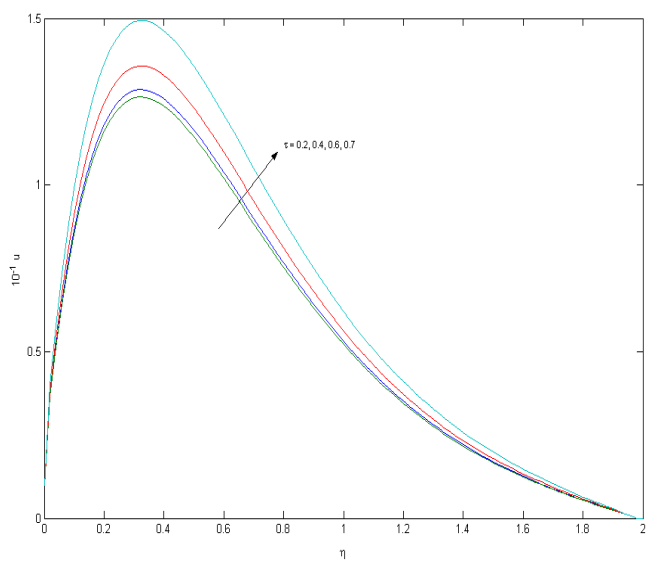

Figure 6: Velocity profiles for time $\tau$ when $\operatorname{Pr}=7$, $G r=5$ and $R a=1$

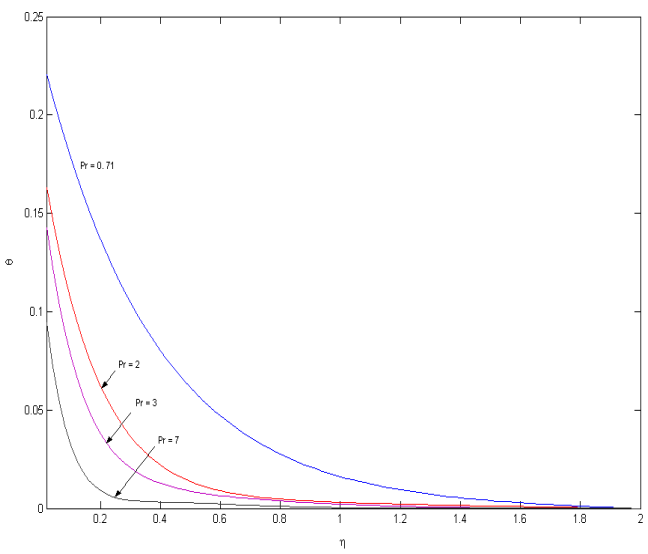

Figure 7: Temperature profiles for $\operatorname{Pr}$ when $R a=5$ and $\tau=0.2$

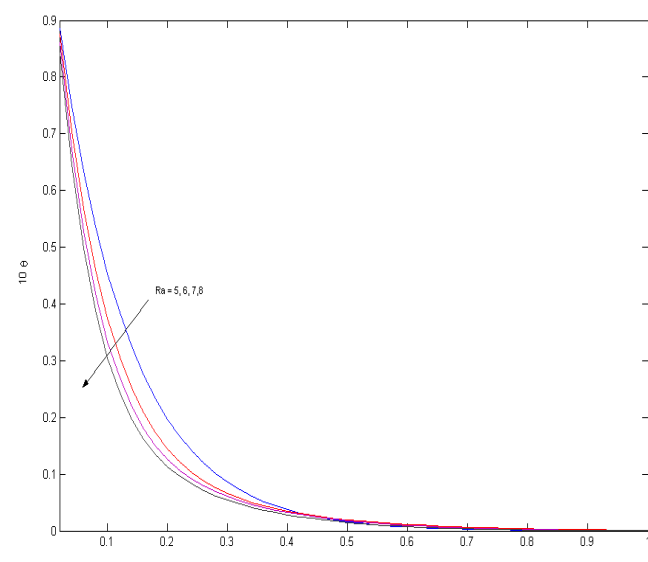

Figure 8: Temperature profiles for $R a$ when $\operatorname{Pr}=7$ and $\tau=\mathbf{0 . 2}$ 


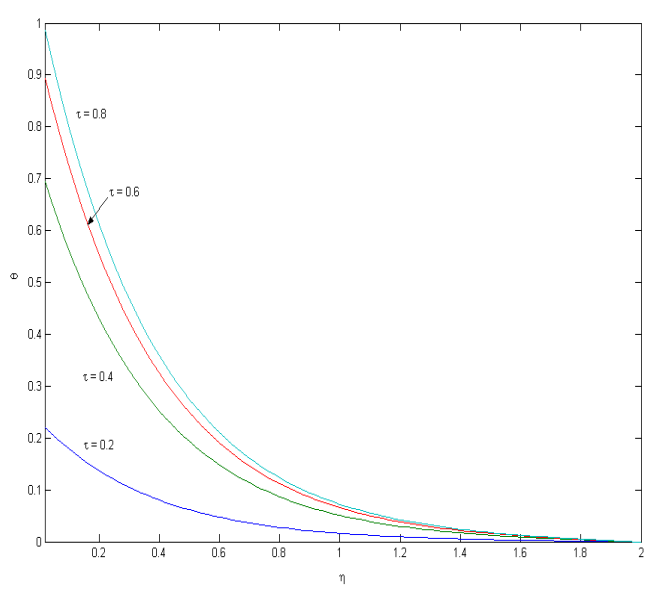

Figure 9: Temperature profiles for time $\tau$ when $P r=0.71$ and $R a=5$

The non-dimensional form of the shear stress $\tau_{x}$ and the rate of heat transfer $-\left(\frac{d \theta}{d \eta}\right)_{\eta=0}$ at the plate $\eta=0$ are given respectively by

$$
\begin{aligned}
& \tau_{x}=\left(\frac{d u}{d \eta}\right)_{\eta=0}, \\
& \text { and }-\left(\frac{d \theta}{d \eta}\right)_{\eta=0}=1+\theta(0, \tau) .
\end{aligned}
$$

Numerical values of the non-dimensional shear stress $\tau_{x}$ and the rate of heat transfer $-\left(\frac{d \theta}{d \eta}\right)_{\eta=0}$ at the plate $\eta=0$ due to the flow are presented in Tables 1 and 2 for several values of Prandtl number $P r$, radiation parameter $R a$ and time $\tau$ with $G r=5$. It is seen from Table 1 that the shear stress $\tau_{x}$ at the plate $\eta=0$ decreases with an increase in either Prandtl numbe $\mathrm{Pr}$ or radiation parameter $R a$ while it increases with an increase in time $\tau$. Further, it is seen from Table 2 that the rate of heat transfer $-\left(\frac{d \theta}{d \eta}\right)_{\eta=0}$ decreases with an increase in either Prandtl numbe $P r$ or $R a$ while it increases with an increase in time $\tau$.

Table 1. Shear stress $10^{-3} \tau_{x}$ at the plate $\eta=0$ for $G r=5$

\begin{tabular}{|c|c|c|c|c|c|c|c|c|}
\hline & \multicolumn{4}{|c|}{$P r$} & \multicolumn{4}{c|}{$R a$} \\
\hline$\tau$ & 0.71 & 2 & 3 & 7 & 4 & 6 & 8 & 10 \\
\hline 0.02 & 0.45755 & 0.42889 & 0.40826 & 0.38912 & 0.43377 & 0.40953 & 0.38692 & 0.36554 \\
0.04 & 0.49795 & 0.46823 & 0.44847 & 0.42784 & 0.49006 & 0.47660 & 0.46189 & 0.44951 \\
0.06 & 0.51500 & 0.48499 & 0.46577 & 0.44484 & 0.51834 & 0.50979 & 0.49982 & 0.49299 \\
0.08 & 0.52291 & 0.49289 & 0.47401 & 0.45305 & 0.53423 & 0.52864 & 0.51998 & 0.51880 \\
\hline
\end{tabular}

Table 2. Rate of heat transfer $-\left(\frac{d \theta}{d \eta}\right)_{\eta=0}$ at the plate $\eta=0$

\begin{tabular}{|c|l|c|c|c|c|c|c|c|}
\hline & \multicolumn{4}{|c|}{$\operatorname{Pr}$ with $R a=2$} & \multicolumn{4}{c|}{$\operatorname{Ra}$ with $\operatorname{Pr}=0.71$} \\
\hline$\tau$ & 0.71 & 2 & 3 & 7 & 2 & 4 & 6 & 8 \\
\hline 0.02 & 1.24905 & 1.23915 & 1.21281 & 1.12955 & 1.24905 & 1.69905 & 1.65221 & 1.58861 \\
0.04 & 2.19761 & 1.54160 & 1.40786 & 1.23688 & 2.19761 & 2.04700 & 1.83529 & 1.69019 \\
0.06 & 2.76211 & 1.68497 & 1.50553 & 1.28675 & 2.76211 & 2.22176 & 1.89948 & 1.71445 \\
0.08 & 3.19385 & 1.77643 & 1.56487 & 1.31502 & 3.19385 & 2.31999 & 1.92399 & 1.72069 \\
\hline
\end{tabular}

\section{CONCLUSION}

The unsteady free convection flow with radiative heat transfer of a viscous incompressible fluid past an impulsively started infinite vertical plate with Newtonian heating has been investigated. It is found that the fluid velocity $u$ decreases near the plate and it increases away from the plate with an increase in either Prandtl number $P r$ or radiation parameter $R a$. The fluid velocity increases with an increase in either Grashof number $G r$ or time $\tau$. It is also found that the fluid temperature $\theta$ decreases with an increase in either Prandtl number $\operatorname{Pr}$ or radiation parameter $R a$. Further, it is found that the shear stress $\tau_{x}$ at the plate $(\eta=0)$ due to the flow decreases with an increase in either radiation parameter $R a$ or
Prandtl number $P r$. The rate of heat transfer $-\left(\frac{d \theta}{d \eta}\right)_{\eta=0}$ decreases with an increase in either Prandtl number $\operatorname{Pr}$ or $R a$ radiation parameter $R a$ while it increases with an increase in time $\tau$.

\section{REFERENCES}

[1] Korycki, R. (2006). Sensitivity analysis and shape optimization for transient heat conduction with radiation. Int. J. Heat Mass Transfer. 49: 2033-2043.

[2] Hottel, H. C. and Sarofim, A. (1967). Radiation Heat Transfer. MacGraw Hill. 
[3] Brewster, M. Q. (1992). Thermal Radiative transfer and Properties. New York: John Wiley and Sons, Inc.

[4] Cess, R.D. (1966). The interaction of therma radiation with free convection heat transfer. Int. J. Heat and Mass transfer. 9:1269-1277.

[5] Merkin, J. H. (1994). Natural convection boundarylayer flow on a vertical surface with Newtonian heating. Int. J. Heat Fluid Flow. 15:392-398.

[6] Hossain, M. A. and Takhar, H. S.(1996). Radiation effect on mixed convection along a vertical plate with uniform surface temperature. Heat amd Mass transfer. 31: 243-248.

[7] Lesnic, D., Ingham, D. B. and Pop, I.(1999): Free convection boundary layer flow along a vertical surface in a porous medium with Newtonian heating. Int. J. Heat Mass Transfer. 42: 2621-2627.

[8] Muthucumaraswamy, R. and Ganesan, P. (2003). Radiation effects on the flow past an impulsively started infinite vertical plate with variable temperature. Int. J. Appl. Mech. Eng. 8(1): 125-129.

[9] Chaudhary, R. C. and Jain, P. (2006). Unsteady free convection boundary-layer flow past an impulsively started vertical surface with Newtonian heating. Rom. Journ. Phys. 51(9-10): 911-925.

[10] Chaudhary, R. C. and Jain, P. (2007). An exact solution to the unsteady free-convection boundary-layer flow past an impulsively started vertical surface with
Newtonian heating. J.Eng. Phys. Thermophys. 80: 954960.

[11] Mebine, P. and Adigio, E. M. (2009). Unsteady free convection flow with thermal radiation past a vertical porous plate with Newtonian heating. Turk J. Phys. 33: $109-119$.

[12] Narahari, M. and Ishak, A. (2011). Radiation effects on free convection near a moving vertical plate with Newtonian heating. J. Applied Science. 11(7): 1096-1104.

[13] Cogley, A.C., Vincentine, W. C. and Gilles, S.E. (1968). A Differential approximation for radiative transfer in a non-gray gas near equilibrium. AIAA Journal. 6: 551555.

[14] Recktenwald, G. W. (2011). Finite-difference approximations to the heat equation.

[15] Ames, W. F. (1992). Numerical Methods for Partial Differential Equations. Academic Press, Inc., Boston, Third edition.

[16] Isaacson, E. and Keller, H. B. (1994). Analysis of Numerical Methods. Dover, New York.

[17] Burden, R. L. and Faires, J. D. (1997). Numerical Analysis. Brooks/Cole Publishing Co. New York, Sixth edition.

[18] Carnahan, B., Luther, H. A. and Wilkes, J. O. (1969) Applied Numerical Methods. John Wiley \& Sons, New York. 